2003-03-22

\title{
Interaction energy surfaces of small hydrocarbon molecules
}

Richard L. Rowley

rowley@byu.edu

Jukka-Pekka Jalkanen

Tapani A. Pakkanen

Yan Yang

Follow this and additional works at: https://scholarsarchive.byu.edu/facpub

Part of the Chemical Engineering Commons

\section{Original Publication Citation}

J.-P. Jalkanen, T.A. Pakkanen, Y. Yang, and R.L. Rowley, â€œInteraction energy surfaces of small hydrocarbon moleculesâ€, J. Chem. Phys.118, 5474 (23)

\section{BYU ScholarsArchive Citation}

Rowley, Richard L.; Jalkanen, Jukka-Pekka; Pakkanen, Tapani A.; and Yang, Yan, "Interaction energy surfaces of small hydrocarbon molecules" (2003). Faculty Publications. 505.

https://scholarsarchive.byu.edu/facpub/505 


\title{
Interaction energy surfaces of small hydrocarbon molecules
}

\author{
Jukka-Pekka Jalkanen and Tapani A. Pakkanen ${ }^{\text {a) }}$ \\ Department of Chemistry, University of Joensuu, FIN-80101 Joensuu, Finland \\ Yan Yang and Richard L. Rowley \\ Department of Chemical Engineering, Brigham Young University, Provo, Utah 84602
}

(Received 12 August 2002; accepted 3 December 2002)

\begin{abstract}
Nonbonding interactions of small alkane molecules were studied with ab initio methods. Previously reported energy data for ethane and propane dimers were supplemented with 1747 new configurations of dimers involving slightly larger hydrocarbons. The completed work provides interaction energy surfaces for all combinations of dimer pairs involving ethane, propane, isobutane, and neopentane and thus contains information of all chemical groups found in acyclic alkanes. The strongest attraction of the studied molecule pairs was encountered in isobutane $\mathrm{C}_{2 h}$ dimer, where an energy minimum of $-1.784 \mathrm{kcal} \mathrm{mol}^{-1}$ at $4.28 \AA$ separation of centermost carbon atoms was observed. The composite data set was fitted with a modified Morse pair potential energy function representing each interatomic $\mathrm{C}-\mathrm{C}, \mathrm{C}-\mathrm{H}$, and $\mathrm{H}-\mathrm{H}$ interaction for easy transfer to molecular dynamics simulation programs. The new generic parameter set was shown to describe the ab initio data for these small alkane molecules with good accuracy. Qualitative comparisons with previously reported potential models were also made, and the relative capability of the models to reproduce quantum-chemical potential energy surfaces was investigated. (C) 2003 American Institute of Physics. [DOI: $10.1063 / 1.1540106]$
\end{abstract}

\section{INTRODUCTION}

Accurate description of dispersion interactions is challenging because their effects are often masked by stronger Coulombic forces. Noble gases and saturated hydrocarbons are good examples of molecules in which there are no strong polar interactions permitting direct determination of the weaker van der Waals forces. Electron correlation between these molecules produces a temporary fluctuation of charge distribution, which is primarily responsible for the attraction of hydrocarbon molecules. This spatial distribution of electrons changes as other atoms come in close proximity, and it depends on the number and type of interacting atoms. The dispersion energy is therefore dependent on the relative orientation of the interacting molecules, as illustrated in our earlier work ${ }^{1}$ on propane dimers. This orientational dependence is missed if the molecular potential surface is probed with a single atom, e.g., with a noble gas atom. While a probe-atom approach reduces the spatial dimensionality of the problem, there is no guarantee that the resulting description of the interactions represents those between two hydrocarbon molecules. We choose instead to use pairs of the test molecules in order to avoid assumptions about the nature of the probe-molecule interaction and its relationship to the true dimer interaction.

Intermolecular phenomena can be studied experimentally, for example, by studying crystal structures ${ }^{2,3}$ or using spectroscopic methods (see, e.g., Ref. 4, and references therein). A popular computational approach to this problem has been to use Monte Carlo ${ }^{5-10}$ or molecular dynamics simulations ${ }^{11}$ to compute macroscopic properties based on

${ }^{\text {a)} E l e c t r o n i c ~ m a i l: ~ t a p a n i . p a k k a n e n @ j o e n s u u . f i ~}$ pair potential models. In both of these methods, the pair potential parameters are adjusted to reproduce experimental measurements of thermophysical properties or vapor-liquid coexistence data. However, these methods produce averaged or indirect information of effective multibody potentials that can be density dependent. Building an accurate model describing the multidimensional energy surfaces is difficult if based solely on this data. Ab initio methods offer a detailed, rigidly controlled approach to study these effects. Using quantum chemistry, one can study the potential energy surfaces of varying sizes at an arbitrary orientation yielding a more comprehensive picture of phenomena arising from intermolecular interactions. In contrast to simulation methods, the quantum-chemistry approach yields a true pair potential consisting only of interactions between two molecules. This provides a clear opportunity to eventually add multibody interactions in a systematic way and eliminate effective potentials that are state dependent.

In the last few years, $a b$ initio studies of weak, nonbonding intermolecular potentials of various hydrocarbon molecules have been published. The rapid increase in computer power has facilitated the study of various hydrocarbon systems ranging from small alkanes ${ }^{1,12-27}$ and acyclic $\pi$-electron systems ${ }^{19-21,28-32}$ to aromatic rings up to anthracene, ${ }^{28,32-39}$ but most studies of larger molecules are still restricted to highly symmetric orientations in which the use of symmetry decreases the computational cost significantly. In this article we present potential energy surfaces of isobutane and neopentane dimers and their combinations with each other as well as ethane and propane. We extend the work done previously with ethane ${ }^{25}$ and propane ${ }^{1}$ dimers to cover ten different molecule pairs. Our aim is to create a 
TABLE I. Isobutane structural parameters.

\begin{tabular}{lccccc}
\hline \hline Bond & Bond length $(\AA)$ & Angle & Angle $(\mathrm{deg})$ & Dihedral & Dihedral angle $(\mathrm{deg})$ \\
\hline $\mathrm{C} 3-\mathrm{C} 1$ & 1.5233 & $\mathrm{C} 3-\mathrm{C} 1-\mathrm{Ha}$ & 108.3016 & $\mathrm{Hb}-\mathrm{C} 3-\mathrm{C} 1-\mathrm{Ha}$ & 60.409 \\
$\mathrm{Hc}-\mathrm{C} 3$ & 1.0915 & $\mathrm{Hb}-\mathrm{C} 3-\mathrm{C} 1$ & 111.3894 & $\mathrm{Hc}-\mathrm{C} 3-\mathrm{C} 1-\mathrm{Ha}$ & 180.0 \\
$\mathrm{Hb}-\mathrm{C} 3$ & 1.0898 & $\mathrm{Hc}-\mathrm{C} 3-\mathrm{C} 1$ & 110.0835 & Point group & $\mathrm{C} 3 \mathrm{v}$ \\
$\mathrm{Ha}-\mathrm{C} 1$ & 1.0935 & & & & \\
\hline \hline
\end{tabular}

general potential model, which is suitable for description of nonbonding interactions of alkanes. We report 1747 new configurations and regress a simple pair potential model that is able to reproduce our ab initio results with good accuracy.

\section{COMPUTATIONAL DETAILS}

\section{A. Selected data set}

Our work concentrated on interactions between small saturated hydrocarbons. In particular, we have computed energies for all the pair combinations of neopentane, isobutane, propane, and ethane (NIPE) molecules as a function of position and relative orientation. We do this with the intent of representing the resultant spatially complex potential energy surface as a sum of simple, spherically symmetric, interatomic potential functions. Each atomic potential center is assumed to be located at the center of the atom and to be unique for each bond environment. Thus, in a $-\mathrm{CH}_{x}$ group $(x$ ranging from 0 to 3 ) there can be three types of $\mathrm{H}$ centers and four types of $\mathrm{C}$ centers based on the bond environment. If one excludes induction effects from neighboring atoms, the ten NIPE dimer combinations represent the smallest collection of hydrocarbons that contain complete information about all of the interatomic interactions found in alkanes. A more detailed description of the types of different interactions is described in Fig. 1 of the Supplementary Data section of Ref. 40.

\section{B. Method and basis set}

The selection of computational method is crucial for obtaining the most reliable description of intermolecular inter-

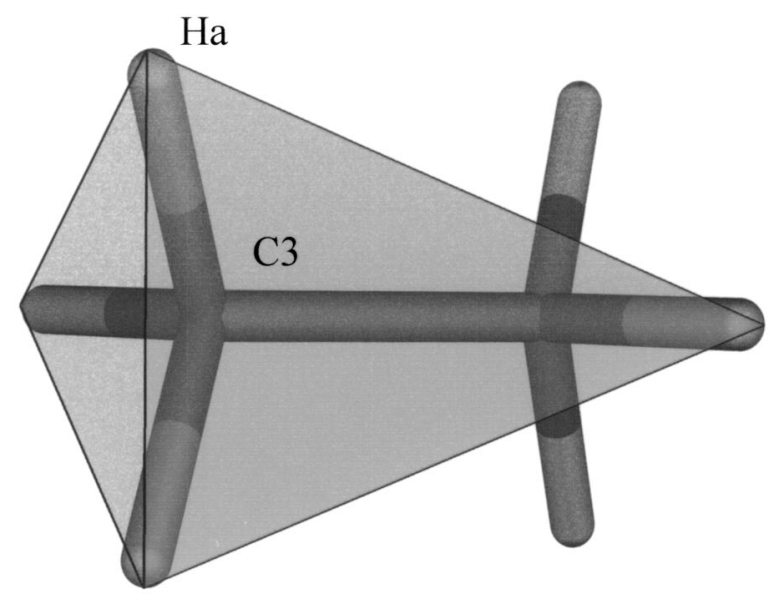

FIG. 1. Ethane (E) monomer labeling. Faces and edges are named after their vertices. The Eaas edge is between the two faces, the Eaal-edge is between hydrogen atoms on the different methyl group. actions of alkanes. ${ }^{40}$ Various authors have reported information on the performance of different computational methods and basis sets. Description of dispersion interactions with Hartree-Fock and density functional methods has been shown to be insufficient, ${ }^{15,19,20,41,42}$ and a more rigorous approach is necessary. Capturing the dispersion interactions of hydrocarbons requires the inclusion of electron correlation, the genesis of dispersion attractions. The present work was done with the $6-311+\mathrm{G}(2 d f, 2 p d)$ basis set and second order Møller-Plesset perturbation theory (MP2) using both GAUSSIAN $94^{43}$ and GAUSSIAN $98 .{ }^{44}$ At the MP2 level, most of the correlation effects are accounted for and introduction of computationally heavier methods (MP4(SDTQ), CCSD(T), CISD) do not significantly affect the intermolecular attraction of alkanes. ${ }^{19,20,23,26,41}$ While MP2 produces a good description of alkane intermolecular behavior it reportedly overestimates the attraction of some aromatic systems. ${ }^{28,38,45,46}$ However, use of electron correlation introduces a strong dependency between the resultant interaction energy and the basis set size. Several papers address the effect of basis set size on interaction energy used in conjunction with various electron correlation methods. ${ }^{19-21,23,26,41}$ Counterpoise correction ${ }^{47}$ was applied to all computed dimer interaction energies to account for basis set superposition error.

\section{Molecular geometries}

Molecular geometries were obtained by running MP2/6-311+ G( $2 d f, 2 p d)$ optimization of all hydrocarbon monomers studied. These fixed geometries, electron correlation method, and basis set were used throughout this work for all dimer calculations. Structural properties of isobutane and neopentane are given in Tables I and II. The same ethane and propane structures were used as described in Refs. 1 and 25. For ease of discussion of different dimer orientations, each molecule is divided into vertices, edges, and faces. These geometrical constructs, defined in Figs. 1-4, involve points, lines, and planes defined by the outermost hydrogen atoms in the molecules. The different combinations of faces, edges, and vertices are designated as main routes.

TABLE II. Neopentane structural parameters.

\begin{tabular}{cccccc}
\hline \hline & $\begin{array}{c}\text { Bond } \\
\text { length } \\
(\AA)\end{array}$ & Angle & $\begin{array}{c}\text { Angle } \\
(\mathrm{deg})\end{array}$ & Dihedral & $\begin{array}{c}\text { Dihedral } \\
\text { angle } \\
(\mathrm{deg})\end{array}$ \\
\hline $\mathrm{C} 3-\mathrm{C} 0$ & 1.5257 & $\mathrm{C} 3-\mathrm{C} 0-\mathrm{C} 3$ & 109.4712 & $\mathrm{C} 3-\mathrm{C} 0-\mathrm{C} 3-\mathrm{C} 3$ & 120.0 \\
$\mathrm{C} 3-\mathrm{Ha}$ & 1.0908 & $\mathrm{Ha}-\mathrm{C} 3-\mathrm{C} 0$ & 110.8753 & $\mathrm{Ha}-\mathrm{C} 3-\mathrm{C} 0-\mathrm{C} 3$ & 60.0 \\
& & & & Point group & $T d$ \\
\hline \hline
\end{tabular}




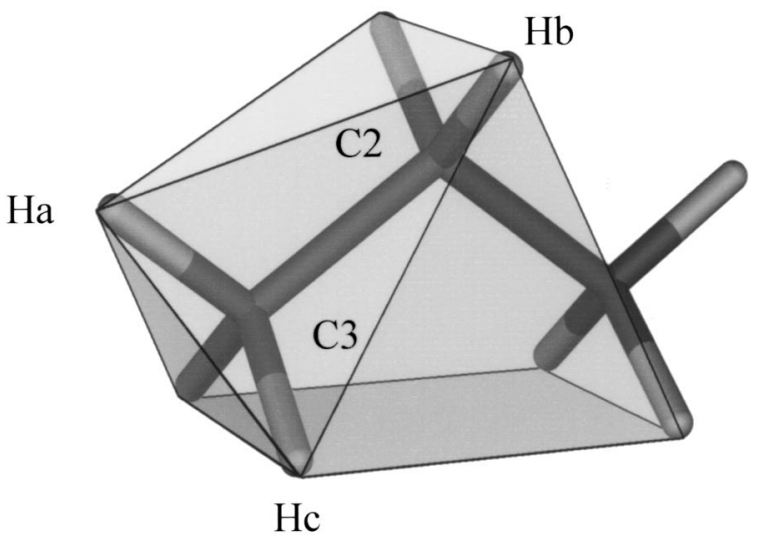

FIG. 2. Propane (P) monomer labeling. Faces and edges are named after their vertices. Pab edge is between Pabc and Pabb faces, Pac edge between $\mathrm{Pabc}$ and Pacc faces, Pbc edge between Pbcc and Pabc faces, Pbb edge is between the two $\mathrm{Hb}$ atoms, Pccs-edge is between the two Hc atoms on the same methyl group, and Pccl-edge is between the two Hc atoms on different methyl groups.

The first letter of any route name designates the molecule and subsequent letters identify the orientation in terms of these geometrical constructs. In some cases, there is ambiguity between two different planes or edges consisting of the same type of hydrogen atoms. These are differentiated by introducing a letter $\mathrm{s}$ for the small plane (or short in the case of an edge) or 1 for the large plane (or long in the case of an edge) (see Figs. 1-4). A complete scan of the potential energy surface would also include intermolecular rotation of one monomer relative to the other along the approach axis of the two molecules. Rotation of monomer about the approach axis has a large effect on dimer interaction energy for some of the routes studied, but little effect on others. Rotation is included on route names with a number, signaling the intermolecular dihedral angle. As an example of the naming system used in this work we consider the route Iabbs-Iabbs 180. This name corresponds to two small isobutane abb-faces interacting, rotated $180^{\circ}$ about the intermolecular axis. Intermolecular approach axes intercept vertices along the $\mathrm{C}-\mathrm{H}$ bond, edges at the center points of the edge, and faces at the

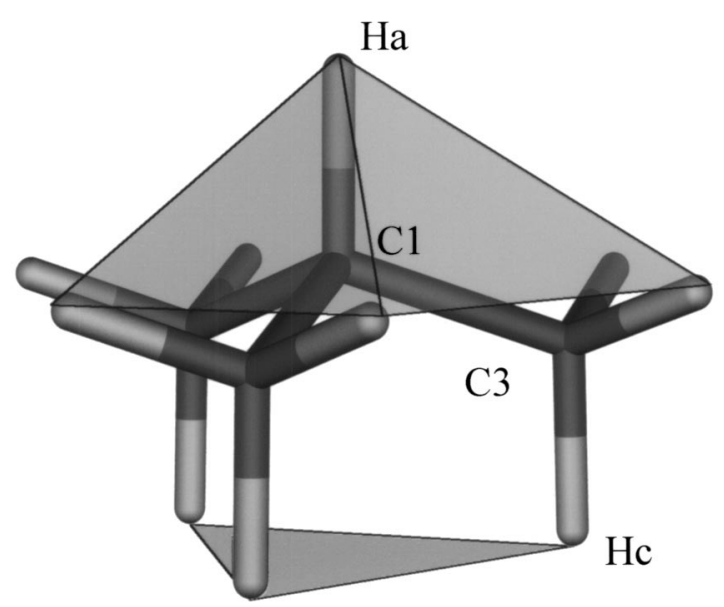

$\mathrm{Hb}$

FIG. 3. Isobutane (I) monomer labeling. Faces and edges are named after their vertices. Iab-edge is between the Iabbl and Iabbs faces.

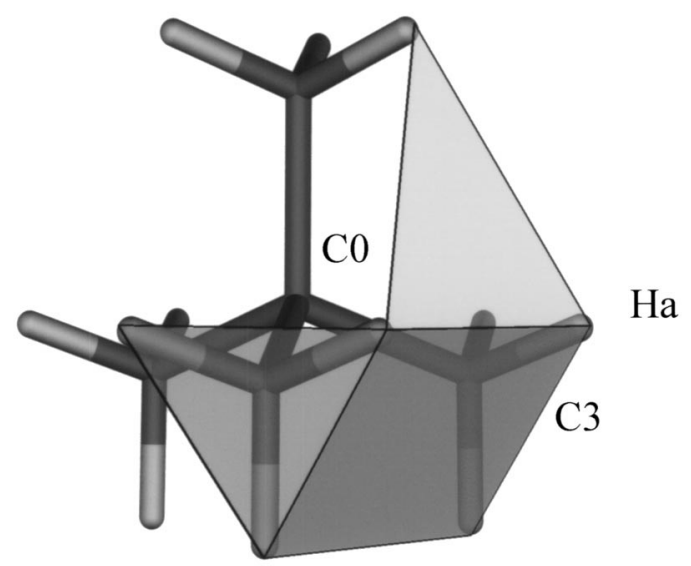

FIG. 4. Neopentane (N) monomer labeling. Faces and edges are named after their vertices. Naas-edge is between Ha atoms on the same methyl group, Naal edge is between Ha atoms on different methyl groups.

midpoint of the vertices forming the face. Faces and edges are held perpendicular to each other. Intermolecular distances in orientations for propane, isobutane, and neopentane are always measured as a separation of centermost carbons. In the case of ethane, distances are measured relative to the center point of the carbon-carbon bond.

\section{RESULTS AND DISCUSSION}

Previously reported data for ethane and propane dimer potential energy surfaces were used in conjunction with the results reported here for configurations of larger alkanes. Propane and ethane dimer data reported in Refs. 1 and 25 were used in conjunction with energy data of other combinations of the NIPE set. To simplify the presentation of a large amount of numerical data describing the calculated potential energy surfaces, each dimer route was fitted to the modified Morse potential,

$$
E=-\epsilon\left[1-\left\{1-\exp ^{-A\left(r_{i j}-r^{*}\right)}\right\}^{2}\right],
$$

independently of each other. This procedure gives an accurate description of the interaction energy curve as a function of intermolecular distance. The complete numerical data set consisting of all calculated dimer energies at various intermolecular distances is available from the authors upon request. Parameters of this fitting are given in Table III. Epsilon in the second column of Table III indicates the depth of the attractive potential well. A large epsilon indicates a large attractive interaction. Parameter $A$ (third column) characterizes the steepness of the potential energy curve where a large value denotes a steep curve. Parameter $r^{*}$ (fourth column) identifies the separation distance at the energy minimum of two molecules at that specific orientation. The fifth column shows the quality of the least-squares fit $\left(\mathrm{kcal} \mathrm{mol}^{-1}\right)^{2}$ of the MP2 data points.

Metzger et al. ${ }^{14}$ used AM1 and PM3 semiempirical methods to study neopentane and methane dimer interaction energies and compared these to MP2/6-311G $(2 d, 2 p) a b$ initio results. Some of the semiempirical methods were found to yield unrealistic behavior as part of the energy curves turned over to less repulsive energies at shorter intermolecu- 
TABLE III. Parameters for $a b$ initio curves, obtained by doing a least-squares fit to Eq. (1). SSR stands for sum of squared residuals in $\left(\mathrm{kcal}^{\mathrm{mol}} \mathrm{m}^{-1}\right)^{2}$.

\begin{tabular}{|c|c|c|c|c|c|c|c|c|c|c|c|c|c|c|}
\hline Route & $\begin{array}{c}\epsilon \\
\left(\mathrm{kcal} \mathrm{mol}^{-1}\right)\end{array}$ & $\left(\begin{array}{c}A \\
\left(\AA^{-1}\right)\end{array}\right.$ & $\begin{array}{l}r^{*} \\
(\AA)\end{array}$ & $\begin{array}{c}\mathrm{SSR} \\
\left(\mathrm{kcal} \mathrm{mol}^{-1}\right)^{2}\end{array}$ & Route & $\begin{array}{c}\epsilon \\
\left(\mathrm{kcal} \mathrm{mol}^{-1}\right)\end{array}$ & $\left(\begin{array}{c}A \\
\left(\AA^{-1}\right)\end{array}\right.$ & $\begin{array}{l}r^{*} \\
(\AA)\end{array}$ & $\begin{array}{c}\mathrm{SSR} \\
\left(\mathrm{kcal} \mathrm{mol}^{-1}\right)^{2}\end{array}$ & Route & $\begin{array}{c}\epsilon \\
\left(\mathrm{kcal} \mathrm{mol}^{-1}\right)\end{array}$ & $\left(\begin{array}{l}A \\
\left(\AA^{-1}\right)\end{array}\right.$ & $\begin{array}{l}r^{*} \\
(\AA)\end{array}$ & $\begin{array}{c}\mathrm{SSR} \\
\left(\mathrm{kcal} \mathrm{mol}^{-1}\right)^{2}\end{array}$ \\
\hline $\mathrm{Pb}-\mathrm{Ea}$ & 0.317 & 1.459 & 5.026 & 0.002 & Naal-Eaal & 1.226 & 1.347 & 4.606 & 0.006 & Naaaa-Pbc & 1.116 & 1.448 & 5.077 & 0.020 \\
\hline $\mathrm{Pb}$-Eaal & 0.747 & 1.484 & 4.090 & 0.022 & Naal-Eaaas & 0.751 & 1.433 & 5.497 & 0.005 & Naaaa-Pbb & 1.052 & 1.343 & 4.601 & 0.008 \\
\hline $\mathrm{Pb}$-Eaaas & 0.518 & 1.453 & 4.845 & 0.002 & Naal-Eaaal & 0.933 & 1.409 & 4.897 & 0.007 & Naaaa-Pb & 1.054 & 1.297 & 4.774 & 0.006 \\
\hline Pab-Ea & 0.596 & 1.405 & 4.486 & 0.001 & Naaal-Eaal & 0.946 & 1.412 & 4.833 & 0.009 & & & & & \\
\hline Pab-Eaal & 1.042 & 1.477 & 3.969 & 0.004 & Naaal-Eaaas & 0.928 & 1.358 & 5.253 & 0.004 & Ia-Ia & 0.685 & 1.401 & 4.499 & 0.008 \\
\hline Pab-Eaaas & 0.651 & 1.520 & 4.756 & 0.004 & Naaal-Eaaal & 1.124 & 1.365 & 4.695 & 0.007 & Ia-Ia 180 & 0.694 & 1.399 & 4.495 & 0.008 \\
\hline Pbb-Ea & 0.442 & 1.459 & 4.624 & 0.001 & Naaaa-Eaal & 1.076 & 1.369 & 4.729 & 0.008 & Iccc-Iccc 0 & 0.969 & 1.443 & 5.691 & 0.026 \\
\hline Pbb-Eaal & 0.581 & 1.411 & 4.136 & 0.003 & Naaaa-Eaaas & 0.752 & 1.439 & 5.449 & 0.004 & Iccc-Iccc 180 & 1.298 & 1.240 & 5.334 & 0.004 \\
\hline Pbb-Eaaas & 0.522 & 1.504 & 4.631 & 0.003 & Naaaa-Eaaal & 0.976 & 1.408 & 4.875 & 0.007 & Ia-Icce 0 & 1.379 & 1.264 & 4.541 & 0.008 \\
\hline Pabb-Ea & 0.568 & 1.358 & 4.483 & 0.001 & & & & & & Ia-Iccc 180 & 1.335 & 1.224 & 4.533 & 0.006 \\
\hline Pabb-Eaal & 1.062 & 1.466 & 3.910 & 0.004 & $\mathrm{~Pb}-\mathrm{Ia}$ & 0.540 & 1.422 & 4.562 & 0.009 & Iab-Iab 0 & 1.135 & 1.440 & 4.356 & 0.017 \\
\hline \multirow[t]{2}{*}{ Pabb-Eaaas } & 0.681 & 1.510 & 4.700 & 0.004 & Pbb-Ia & 0.707 & 1.416 & 4.182 & 0.010 & Iab-Iab 90 & 1.458 & 1.462 & 4.238 & 0.028 \\
\hline & & & & & Pab-Ia & 1.163 & 1.441 & 4.112 & 0.013 & Iabbs-Iabbs 0 & 0.968 & 1.473 & 4.558 & 0.014 \\
\hline Ea-Ia & 0.429 & 1.498 & 4.931 & 0.008 & Pabb-Ia & 0.984 & 1.393 & 3.973 & 0.010 & Iabbs-Iabbs 180 & 1.309 & 1.570 & 4.725 & 0.024 \\
\hline Ea-Iab & 0.759 & 1.405 & 4.500 & 0.005 & $\mathrm{~Pb}-\mathrm{Iab}$ & 0.987 & 1.407 & 4.206 & 0.011 & Iabbl-Iabbl 0 & 1.240 & 1.483 & 4.301 & 0.017 \\
\hline Ea-Iabbs & 0.706 & 1.445 & 4.655 & 0.007 & Pbb-Iab & 0.849 & 1.408 & 4.186 & 0.012 & Iabbl-Iabbl 180 & 1.784 & 1.493 & 4.282 & 0.033 \\
\hline Ea-Iabbl & 0.908 & 1.360 & 4.335 & 0.005 & Pab-Iab & 1.025 & 1.538 & 4.578 & 0.023 & & & & & \\
\hline Ea-Iccc & 0.723 & 1.279 & 5.085 & 0.002 & Pabb-Iab & 1.021 & 1.546 & 4.257 & 0.021 & Naaaa-Ia & 1.348 & 1.281 & 4.702 & 0.009 \\
\hline Eaas-Ia & 0.559 & 1.427 & 4.871 & 0.004 & Pb-Iabbs & 0.858 & 1.429 & 4.441 & 0.011 & Naaaa-Iab & 1.183 & 1.410 & 5.114 & 0.016 \\
\hline Eaas-Iab & 0.687 & 1.506 & 4.864 & 0.010 & Pbb-Iabbs & 0.838 & 1.462 & 4.307 & 0.010 & Naaaa-Iabbs & 1.114 & 1.451 & 5.207 & 0.019 \\
\hline Eaas-Iabbs & 0.681 & 1.502 & 4.970 & 0.007 & Pab-Iabbs & 0.954 & 1.476 & 4.354 & 0.014 & Naaaa-Iabbl & 1.381 & 1.411 & 4.985 & 0.024 \\
\hline Eaas-Iabbl & 0.865 & 1.453 & 4.689 & 0.009 & Pabb-Iabbs & 0.863 & 1.591 & 4.510 & 0.019 & Naaal-Ia & 1.446 & 1.268 & 4.556 & 0.010 \\
\hline Eaas-Iccc & 0.761 & 1.350 & 5.320 & 0.005 & & & & & & Naaal-Iab & 1.290 & 1.368 & 4.953 & 0.015 \\
\hline Eaal-Ia & 0.910 & 1.393 & 4.064 & 0.007 & Naal-Pabc & 0.952 & 1.447 & 5.274 & 0.014 & Naaal-Iabbs & 1.112 & 1.391 & 5.197 & 0.015 \\
\hline Eaal-Iab & 1.231 & 1.558 & 2.801 & 0.014 & Naal-Pbcc & 1.186 & 1.458 & 5.053 & 0.023 & Naaal-Iabbl & 1.138 & 1.399 & 5.110 & 0.017 \\
\hline Eaal-Iabbs & 0.901 & 1.525 & 4.430 & 0.014 & Naal-Pabb & 1.125 & 1.360 & 4.683 & 0.011 & Naaal-Ia & 1.103 & 1.262 & 4.854 & 0.007 \\
\hline Eaal-Iabbl & 1.070 & 1.505 & 4.184 & 0.023 & Naal-Pab & 0.772 & 1.458 & 5.240 & 0.014 & Naal-Iab & 1.018 & 1.430 & 5.244 & 0.018 \\
\hline Eaal-Iccc & 0.933 & 1.426 & 4.816 & 0.016 & Naal-Pbc & 0.871 & 1.437 & 5.249 & 0.015 & Naal-Iabbs & 1.065 & 1.431 & 5.285 & 0.016 \\
\hline Eaaas-Ia & 0.655 & 1.453 & 4.789 & 0.006 & Naal-Pbb & 0.766 & 1.394 & 4.947 & 0.010 & Naal-Iabbl & 1.301 & 1.402 & 5.019 & 0.021 \\
\hline Eaaas-Iab & 0.795 & 1.481 & 4.789 & 0.007 & Naal-Pb & 0.862 & 1.285 & 4.926 & 0.004 & & & & & \\
\hline Eaaas-Iabbs & 0.703 & 1.558 & 4.969 & 0.010 & Naaal-Pabc & 1.018 & 1.460 & 5.159 & 0.020 & Naal-Naal 0 & 0.878 & 1.351 & 6.034 & 0.011 \\
\hline Eaaas-Iabbl & 0.840 & 1.482 & 4.777 & 0.011 & Naaal-Pbcc & 1.133 & 1.444 & 4.996 & 0.023 & Naal-Naal 90 & 1.456 & 1.228 & 5.417 & 0.011 \\
\hline Eaaas-Iccc & 0.761 & 1.383 & 5.392 & 0.008 & Naaal-Pabb & 1.016 & 1.437 & 4.777 & 0.019 & Naas-Naas 0 & 0.603 & 1.477 & 6.765 & 0.005 \\
\hline Eaaal-Ia & 1.002 & 1.409 & 4.024 & 0.010 & Naaal-Pab & 1.040 & 1.419 & 4.905 & 0.015 & Naas-Naas 90 & 0.669 & 1.449 & 6.694 & 0.005 \\
\hline Eaaal-Iab & 0.877 & 1.471 & 4.345 & 0.009 & Naaal-Pbc & 1.197 & 1.429 & 4.925 & 0.022 & Naaal-Naaal 0 & 1.042 & 1.392 & 5.741 & 0.033 \\
\hline Eaaal-Iabbs & 0.793 & 1.540 & 4.506 & 0.012 & Naaal-Pbb & 1.053 & 1.341 & 4.602 & 0.011 & Naaal-Naaal 60 & 1.441 & 1.234 & 5.351 & 0.014 \\
\hline Eaaal-Iabbl & 1.009 & 1.498 & 4.248 & 0.018 & Naaal-Pb & 1.049 & 1.235 & 4.650 & 0.004 & Naaas-Naaas 0 & 0.635 & 1.520 & 6.776 & 0.008 \\
\hline \multirow[t]{4}{*}{ Eaaal-Iccc } & 0.892 & 1.434 & 4.878 & 0.014 & Naaaa-Pabc & 0.989 & 1.428 & 5.228 & 0.011 & Naaas-Naaas 60 & 0.683 & 1.482 & 6.742 & 0.021 \\
\hline & & & & & Naaaa-Pbcc & 1.260 & 1.404 & 4.966 & 0.017 & Naaaa-Naaaa 0 & 1.288 & 1.329 & 5.679 & 0.023 \\
\hline & & & & & Naaaa-Pabb & 1.008 & 1.445 & 4.830 & 0.016 & Naaaa-Naaaa 90 & 1.014 & 1.389 & 5.890 & 0.026 \\
\hline & & & & & Naaaa-Pab & 1.170 & 1.360 & 4.797 & 0.010 & $\mathrm{Na}-\mathrm{Na}$ & 0.692 & 1.286 & 5.333 & 0.009 \\
\hline
\end{tabular}


lar distances, but the ab initio curves did not have this problem. Metzger et al. reported an energy minimum of -1.361 $\mathrm{kcal} \mathrm{mol}^{-1}$ for a neopentane dimer corresponding to our Naaal-Naaal 60 route. Our calculations show a slightly stronger interaction, $-1.441 \mathrm{kcal} \mathrm{mol}^{-1}$, due to the larger basis set used in our study. The equilibrium distance of $\sim 5.35 \AA$ does agree with our results for this dimer.

The most favorable orientations of each molecule pair studied in this work are illustrated in Figs. 5(a)-5(h), and the interaction energies of these configurations are given in Table IV. The strongest attractions are found for routes, where molecules avoid head-on approaches of atoms. The least attraction occurs for routes, where the closest hydrogen atoms are directly facing each other, as in vertex-vertex routes. The strongest attraction of all the data was found for the $C_{2 h}$ symmetric Iabbl-Iabbl 180 route, $1.784 \mathrm{kcal} \mathrm{mol}^{-1}$. According to our calculations, this minimum is reached at intermolecular separation of $4.28 \AA$. The most attractive orientations for ethane, propane, isobutane, and neopentane paired with neopentane reveal an interesting trend. The deepest minima are $-1.226 \mathrm{kcal} \mathrm{mol}^{-1}$ (Naal-Eaal), -1.260 $\mathrm{kcal} \mathrm{mol}^{-1}$ (Naaaa-Pbcc), - $1.446 \mathrm{kcal} \mathrm{mol}^{-1}$ (Naaal-Ia), and $-1.456 \mathrm{kcal} \mathrm{mol}^{-1}$ (Naal-Naal 90), respectively. The attraction increases with molecular size, but the increment from isobutane-neopentane to the neopentane dimer is not large, only $\sim 0.01 \mathrm{kcal} \mathrm{mol}^{-1}$. The globular shaped neopentane with its protruding methyl groups prevents the closer, more energetically favorable interactions of more open molecules. Although all the neopentane dimer routes studied show attractive potential wells, isobutane interactions with neopentane are more favorable.

\section{A. Fitting the potential energy surfaces with interatomic pair potentials}

A major objective of this and our previous calculations of dimer energy surfaces is the representation of alkane intermolecular potentials as a sum of atomic pair potentials. The hope is that these interatomic pair potentials are transferable and can then be used to adequately describe the intermolecular interactions in molecular dynamics simulations. Several different types of potential energy functions have been investigated previously ranging from a simple LennardJones 12-6 model to more complicated functions. These previous studies have shown that a Morse-type function can be used to accurately describe the interactions at the repulsive region as well as the bottom of the potential well. ${ }^{12,25,48,49} \mathrm{~A}$ Morse-type function has good flexibility as the slope of the repulsion can be adjusted, instead of having a fixed repulsive exponent as in the case of the Lennard-Jones-type function.

To obtain a generic parameter set for alkanes, a sum of pair potentials each in the form of Eq. (1) were fitted to our $a b$ initio data with an unweighted least-squares method. A simulated annealing algorithm by Goffe et al. was used for this purpose. Details of the algorithm can be found elsewhere. ${ }^{1,50}$ Five different fitting runs were conducted, and each of these was independent of the others using random starting points and different initial search directions. The generic parameter set obtained for small alkanes is given in Table V, along with previously reported ethane ${ }^{25}$ and propane $^{1}$ parameter sets. Repulsive interaction energies larger than $\sim 3 \mathrm{kcal} \mathrm{mol}^{-1}$ were not included in the regression to avoid fitting the repulsive side of the potential at the expense of the attractive well. Although simulated annealing is a stochastic global optimization method and covers only a part of the total parameter space, all five runs converged to the same solution. Only slight variations, $\sim 1 \times 10^{-5}$ $\mathrm{kcal} \mathrm{mol}^{-1}$ in $\epsilon_{\mathrm{CC}}$ and $\sim 2 \times 10^{-5} \AA^{-1}$ in $A_{\mathrm{CC}}$, were observed between the different fitting runs. These variations of parameters produced a sum of squared residuals (SSR) of $\sim 3 \times 10^{-8}\left(\mathrm{kcal} \mathrm{mol}^{-1}\right)^{2}$ for the overall fit. We also found that a slight change in the carbon-carbon interaction parameters had a relatively small change in overall quality of the fit, because of the small number of interactions between carbon atoms in the dimers compared to the number of other type interactions. For example, the neopentane dimer has 25 carbon-carbon, 144 hydrogen-hydrogen, and 120 carbonhydrogen interactions. The parameter $\epsilon$ was constrained to be greater than zero to ensure that potentials would not turn over at short distances.

The overall accuracy of the generic parameter set is excellent, yielding a total error of $50.354\left(\mathrm{kcal} \mathrm{mol}^{-1}\right)^{2}$ and an average of $0.015\left(\mathrm{kcal} \mathrm{mol}^{-1}\right)^{2}$ per data point. This result for the ten different pairs of molecules studied is remarkable since all of the potential energy surfaces have been reduced to three interatomic pair potentials: $\mathrm{C}-\mathrm{C}, \mathrm{C}-\mathrm{H}$, and $\mathrm{H}-\mathrm{H}$. (A more detailed description of the error sum on any particular main route studied is presented in Table I of the Supplementary Data section of Ref. 40). Table VI shows the fitting error for each pair and illustrates how the error is distributed between different combinations of molecules. A slight increase in the error is observed for larger systems. This seems consistent, since even the smallest errors in the regressed parameters are bound to accumulate with increasing numbers of interactions. Furthermore, it can be seen from Table VI that the error for the repulsive data points constitute almost twothirds of the overall SSR, even though the majority of the data points are attractive.

In general, the previously reported ethane dimer data ${ }^{25}$ is reproduced better with the generic set than with parameters regressed from the propane data. An error of 8.19 $\left(\mathrm{kcal} \mathrm{mol}^{-1}\right)^{2}$ was obtained when the propane-specific parameters were used to predict the ethane data, but the error was reduced to $4.5\left(\mathrm{kcal} \mathrm{mol}^{-1}\right)^{2}$ when the generic parameter set was used. These results correspond to 0.021 and 0.011 $\left(\mathrm{kcal} \mathrm{mol}^{-1}\right)^{2}$ error per data point, respectively. Only three routes out of 240 give a SSR per data point over 0.1 $\left(\mathrm{kcal} \mathrm{mol}^{-1}\right)^{2}$. The largest error per data point is encountered in the propane dimer route Pccs-Pccc $\left(0.165 \mathrm{kcal} \mathrm{mol}^{-1}\right)^{2}$. In this case, the predicted potential well is deeper and the repulsion starts at slightly closer distances than the corresponding $a b$ initio results. A similar behavior is observed also for routes Pccc-Pcccc and Naaaa-Naaaa 0, i.e., the predicted attraction is stronger and the repulsion gentler than the $a b$ initio data. It seems that the largest errors are concentrated on routes, where non- $\mathrm{CH}_{3}$ groups interact closely. The 

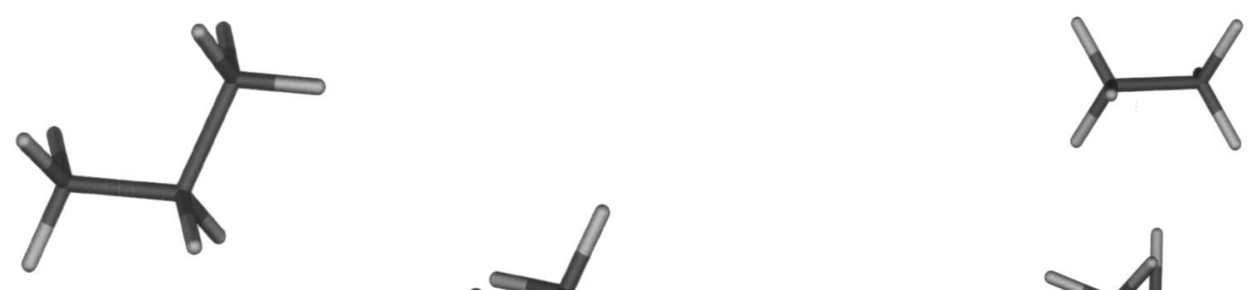

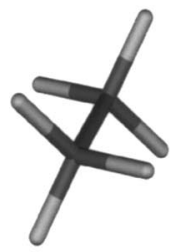

(a) Pabb-Eaal
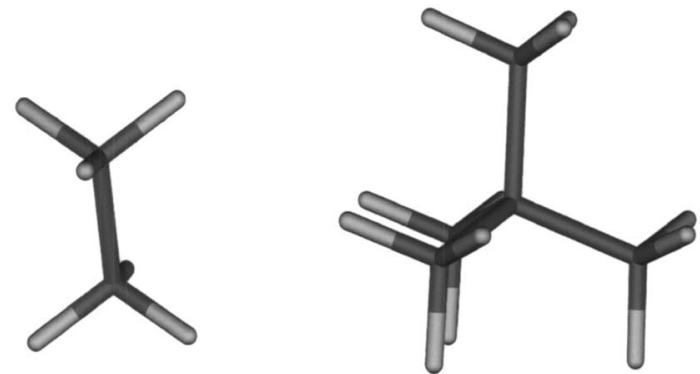

(c) Naal-Eaal

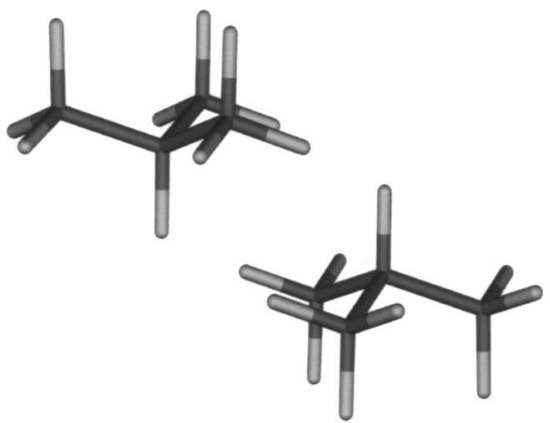

(e) Iabbl-Iabbl
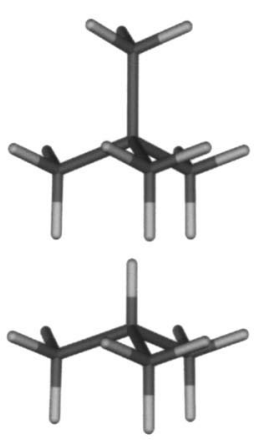

(g) Naaal-Ia

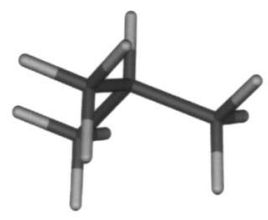

(b) Eaal-Iab
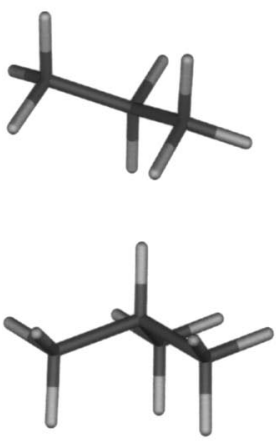

(d) Pab-Ia
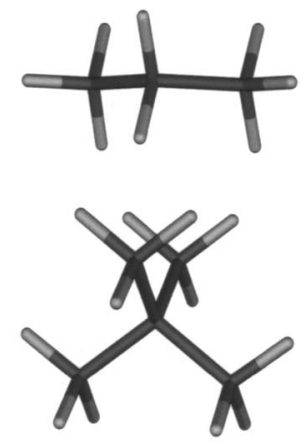

(f) Naaaa-Pbcc
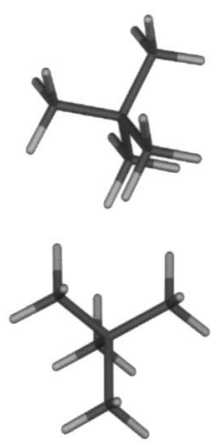

(h) Naal-Naal 90

FIG. 5. (a)-(h) Most attractive orientations of the molecule pairs. The first letter identifies the molecule and the subsequent letters identify the vertex, edge, or face that is held perpendicular to the approach axis.

worst, average, and best-fitted cases of all the calculated alkane routes are depicted in Fig. 6. As can be seen from Fig. 6 , the overall accuracy of the fit is good. The generic parameter set was also used to predict ethane dimer repulsive en- ergies up to several hundred kilocalories. In over half of the repulsive ethane dimer routes it correctly described the dimer behavior, even though energies this high were intentionally excluded from the fitting process. 
TABLE IV. Most favorable orientations of the studied configurations. Well depth in $\mathrm{kcal} \mathrm{mol}^{-1}$. Capital letters designate molecule pair; $\mathrm{E}=$ ethane, $\mathrm{P}=$ propane, $\mathrm{I}=$ isobutane, $\mathrm{N}=$ neopentane. Route column identifies orientation of molecules.

\begin{tabular}{clc}
\hline \hline Molecule pair & \multicolumn{1}{c}{ Route } & $\begin{array}{c}\text { Well depth } \\
\left(\mathrm{kcal} \mathrm{mol}^{-1}\right)\end{array}$ \\
\hline EE $^{\mathrm{a}}$ & Route 19 & 1.046 \\
EI & Eaal-Iab & 1.231 \\
EP & Pabb-Eaal 90 & 1.062 \\
EN & Naal-Eaal & 1.226 \\
IP & Pab-Ia & 1.163 \\
II & Iabbl-Iabbl & 1.784 \\
IN & Naaal-Ia & 1.446 \\
PP & Pbcc-Pbcc 180 & 1.625 \\
PN & Naaaa-Pbcc & 1.260 \\
NN & Naal-Naal 90 & 1.456 \\
\hline \hline
\end{tabular}

${ }^{a}$ Reference 25 .

${ }^{\mathrm{b}}$ Reference 1.

\section{B. Comparison to previous parameter sets}

Parameter sets based only on ethane or propane dimer data were published recently by us. ${ }^{1,25}$ In both of these cases it was found that pair potentials described by the modified Morse function were able to produce energy curves that were in good agreement with $a b$ initio data. The data used to regress the generic parameter set presented in this work included data from our previous work on ethane and propane reported in Refs. 25 and 1. A graphical representation of the different parameter set results is given in Figs. 7(a)-7(c). Locations of potential energy minima on the $x$ axis do not differ much between the Morse sets. Larger differences are observable for the $A$ and $\epsilon$ parameters, which describe the repulsive slope and potential well depth, respectively. The generic carbon-carbon interaction is very similar to that obtained from ethane. The $\mathrm{C}-\mathrm{C}$ potential obtained from propane data is the most attractive of the three Morse potentials. The slope of the $\mathrm{H}-\mathrm{H}$ repulsion is also very similar for all three Morse parameter sets and the size parameter varies only slightly in the order $\mathrm{Er}_{\mathrm{HH}}>\mathrm{Pr}_{\mathrm{HH}}>\mathrm{Gr}_{\mathrm{HH}}$. The generic set $\mathrm{H}-\mathrm{H}$ coincides with the optimized potentials for liquid simulations $^{8}$ (OPLS) $\mathrm{H}-\mathrm{H}$ at $\sim 2 \AA$ separation, but the potential is less attractive at larger distances and less repulsive at shorter distances resulting in "softer" hydrogens. In general, the modified Morse model does not produce as steep a repulsion as does the Lennard-Jones 12-6 potential function.

Cross interactions between carbon and hydrogen atoms are the major source of attraction between alkanes according to all three Morse sets. The ethane parameter set shows the
TABLE VI. Fitting error ((in kcal mol $\left.\left.{ }^{-1}\right)^{2}\right)$ for all molecule pairs. Capital letters identify the interacting molecule pair $(\mathrm{E}=$ ethane, $\mathrm{P}=$ propane, $\mathrm{I}=$ isobutane, $\mathrm{N}=$ neopentane).

\begin{tabular}{lrccc}
\hline \hline & $\begin{array}{c}\text { No. } \\
\text { data } \\
\text { System }\end{array}$ & $\begin{array}{c}\text { Soints, all } \\
\left(\mathrm{kcal} \mathrm{mol}^{-1}\right)^{2}\end{array}$ & $\begin{array}{c}\text { SSR, } \\
\text { attractive points only } \\
\left(\mathrm{kcal} \mathrm{mol}^{-1}\right)^{2}\end{array}$ & $\begin{array}{c}\text { SSR all, } \\
\text { per data point } \\
\left(\mathrm{kcal} \mathrm{mol}^{-1}\right)^{2}\end{array}$ \\
\hline EE & 395 & 4.502 & 1.465 & 0.011 \\
EP & 130 & 2.355 & 0.835 & 0.018 \\
EI & 397 & 3.032 & 1.045 & 0.008 \\
EN & 129 & 1.429 & 0.382 & 0.011 \\
PP & 1245 & 20.293 & 6.521 & 0.016 \\
PI & 206 & 1.078 & 0.267 & 0.005 \\
PN & 330 & 4.748 & 1.570 & 0.014 \\
II & 178 & 3.221 & 0.996 & 0.018 \\
IN & 175 & 3.677 & 1.361 & 0.021 \\
NN & 202 & 6.018 & 2.401 & 0.030 \\
Sum & $\mathbf{3 3 8 7}$ & $\mathbf{5 0 . 3 5 4}$ & $\mathbf{1 7 . 2 4 3}$ & Average: 0.015 \\
\hline \hline
\end{tabular}

strongest $\mathrm{C}-\mathrm{H}$ attraction, but the propane and generic parameters both show considerable attraction. The most striking difference between the models compared here is the difference between the Morse models and the OPLS set which uses a geometric average of the energy parameter and an arithmetic average of the size parameter of the $\mathrm{C}-\mathrm{C}$ and $\mathrm{H}-\mathrm{H}$ interactions to obtain the $\mathrm{C}-\mathrm{H}$ parameters in the Lennard-Jones 12-6 potential. As can be seen in Figs. 7(a)7(c), the generic modified Morse $\mathrm{CH}$ is closer to the OPLS $\mathrm{C}-\mathrm{H}$ potential than the ethane or propane dimer set, but it is still much more attractive at shorter equilibrium distances than the OPLS model potential. Introduction of the $\mathrm{CH}_{2}$ group into the regression through the propane dimer slightly decreases the depth of the attractive well. This might be due to the increased appearance of non- $\mathrm{CH}_{3}$ features in the $a b$ initio data and suggests that even better agreement between the sum of atomic pair interactions and the molecular dimer potential energy surface might be achieved if cross $\mathrm{C}-\mathrm{H}$ interactions between different $\mathrm{CH}_{x}$ groups were to be treated differently, i.e, if the methyl-methylene $\mathrm{C}-\mathrm{H}$ interaction was considered to be different than the methyl-methyl $\mathrm{C}-\mathrm{H}$ interaction.

\section{Comparison to some other potential models}

Some qualitative comparisons of potential energy surfaces produced by the OPLS and ab initio calculations were made to give a general idea of the performance of modified Morse model. Energies of all alkane data points were calculated with the OPLS parameter sets. Both united atom (UA)

TABLE V. Parameter sets for different alkanes. Set labeled as generic includes $a b$ initio data from ethane, propane, isobutane, neopentane, and all their combinations.

\begin{tabular}{|c|c|c|c|c|c|c|c|c|c|}
\hline Set & $\begin{array}{c}\epsilon_{\mathrm{CC}} \\
\left(\mathrm{kcal} \mathrm{mol}^{-1}\right)\end{array}$ & $\begin{array}{l}A_{\mathrm{CC}} \\
\left(\AA^{-1}\right)\end{array}$ & $\begin{array}{l}r_{\mathrm{CC}}^{*} \\
(\AA)\end{array}$ & $\begin{array}{c}\epsilon_{\mathrm{CH}} \\
\left(\mathrm{kcal} \mathrm{mol}^{-1}\right)\end{array}$ & $\begin{array}{l}A_{\mathrm{CH}} \\
\left(\AA^{-1}\right)\end{array}$ & $\begin{array}{l}r_{\mathrm{CH}}^{*} \\
(\AA)\end{array}$ & $\begin{array}{c}\epsilon_{\mathrm{HH}} \\
\left(\mathrm{kcal} \mathrm{mol}^{-1}\right)\end{array}$ & $\begin{array}{l}A_{\mathrm{HH}} \\
\left(\AA^{-1}\right)\end{array}$ & $\begin{array}{l}r_{\mathrm{HH}}^{*} \\
(\AA)\end{array}$ \\
\hline Ethane dimer ${ }^{\mathrm{a}}$ & 0.0689 & 1.3083 & 4.3500 & 0.7409 & 1.8287 & 2.491 & -40.65 & 2.2239 & 0.2456 \\
\hline Propane dimer ${ }^{\mathrm{b}}$ & 0.16105 & 1.2655 & 4.1844 & 0.55162 & 2.2744 & 2.544 & $4.5284 \times 10^{-5}$ & 1.255 & 6.1543 \\
\hline Generic & 0.05133 & 1.45985 & 4.34117 & 0.35562 & 2.11174 & 2.60211 & 0.01048 & 1.26072 & 3.97536 \\
\hline
\end{tabular}

${ }^{\mathrm{a}}$ Reference 25 .

${ }^{\mathrm{b}}$ Reference 1 . 


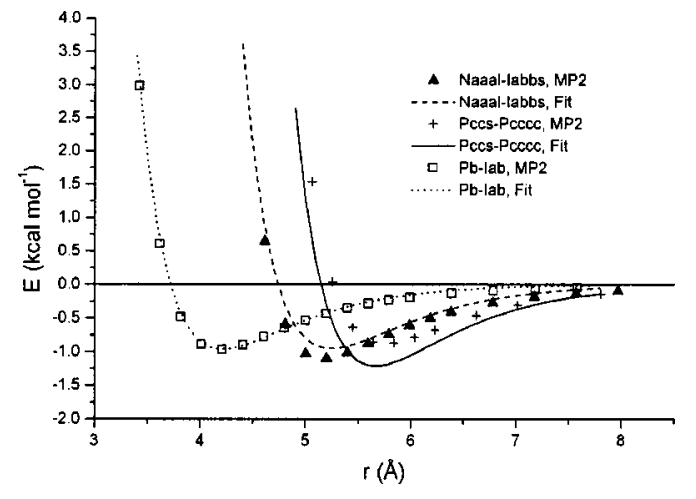

FIG. 6. Quality of fitted potential energy curves. The propane dimer PccsPcccc shows the largest fitting error $\left[\mathrm{SSR}=0.165\left(\mathrm{kcal} \mathrm{mol}^{-1}\right)^{2}\right.$ per data point]. The neopentane-isobutane route Naaal-Iabbs represents an average $\left[\mathrm{SSR}=0.015\left(\mathrm{kcal} \mathrm{mol}^{-1}\right)^{2}\right.$ per data point $]$ error case and the propaneisobutane route $\mathrm{Pb}-\mathrm{Iab}$ is the most accurately $\left(\mathrm{SSR}=0.00047 \mathrm{kcal} \mathrm{mol}^{-1}\right)^{2}$ predicted route. Symbols (diamonds $=$ Pccs-Pcccc, triangles $=$ Naaal-Iabbs, squares $=\mathrm{Pb}-\mathrm{Iab}$ ) represent $a b$ initio $\mathrm{MP} 2$ energies and lines (dashed $=$ Naaal-Iabbs, solid $=$ Pccs-Pcccc, dotted $=\mathrm{Pb}$-Iab) represent energies calculated with the modified-Morse potential model for pairwise interatomic interactions with the generic parameter set.

and all atom (AA) models are based on Monte Carlo simulations of various hydrocarbon properties. The heterogeneous OPLS UA model by Jorgensen et al. ${ }^{5}$ was calibrated to reproduce heats of vaporization and density data for 15 different hydrocarbon liquids consisting of normal and branched alkanes as well as some aromatic and unsaturated molecules. A more detailed OPLS AA model by Kaminski et al. ${ }^{8}$ was regressed from Monte Carlo simulation data of methane, ethane, propane, and butane. Simulated properties included heat of vaporization, molecular volume, heat capacity, the coefficient of thermal expansion, isothermal compressibility, and the $\mathrm{C}-\mathrm{C}$ radial distribution function yielding a good overall estimate of nonbonding properties. Both OPLS models use combining rules to describe the cross interaction between unlike atoms or sites. Both of these parameter sets represent effective pair potentials owing to the use of liquidphase simulations in regression of these sets.

Despite the different approach in potential model construction, potential well depths are reasonably well predicted with the AA model. In many cases, the UA model characterizes the attractive well as too broad and misplaced, although the rough strength of attraction is approximately correct. However, the repulsive side of the potential energy curves is problematic for both models, especially for the UA model. The OPLS-UA model seems to have problems with branched molecules, like isobutane and neopentane, despite the fact that these molecules were part of the original training set used by Jorgensen et al. ${ }^{5}$ In these cases, some of the routes are correctly described, while the bottom of the potential well is too broad. This occurs especially on the routes where two hydrogen atoms are brought close together (e.g., Na-Na, $\mathrm{Pa}-\mathrm{Pa}$, or Ia-Ia). This is understandable, because by definition the UA model does not consider hydrogen atoms separately from the $\mathrm{CH}_{x}$ group.

The AA model is definitely an improvement, but the OPLS AA model exhibits too strong a repulsion on some of the neopentane dimer routes, while the attractive potentials

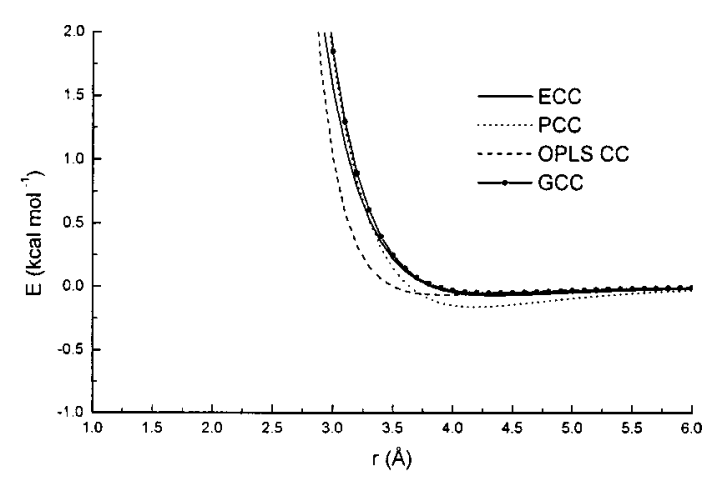

(a) Interatomic interactions for C-C

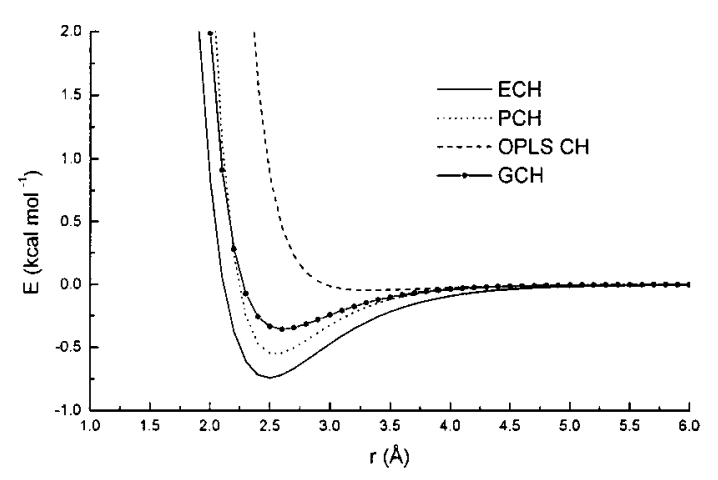

(b) Interatomic interactions for $\mathrm{C}-\mathrm{H}$

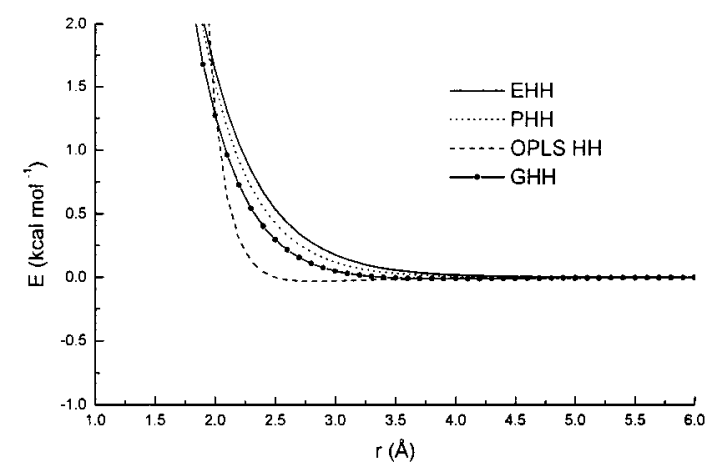

(c) Interatomic interactions for $\mathrm{H}-\mathrm{H}$

FIG. 7. Interatomic interactions for (a) $\mathrm{C}-\mathrm{C}$, (b) $\mathrm{C}-\mathrm{H}$, and (c) $\mathrm{H}-\mathrm{H}$ from different potential models. $\mathrm{E}=$ ethane Morse, $\mathrm{P}=$ propane Morse, $\mathrm{G}=$ generic Morse (NIPE).

are approximately correct, as can be seen in Figs. 8(a)-8(d). Excessive repulsion was observed also with other molecule pairs. Our $a b$ initio results are fairly close to the energy curves produced by the AA model. In general, potential well depths predicted by the AA model are in good agreement with the MP2 energies, but it is surprising to see that in some extreme cases well depths are $80 \%$ larger than the ab initio results. This is a considerably larger difference than the gap between the MP2 $2_{\text {limit }}$ and our calculated values. ${ }^{26}$ Neverthe- 


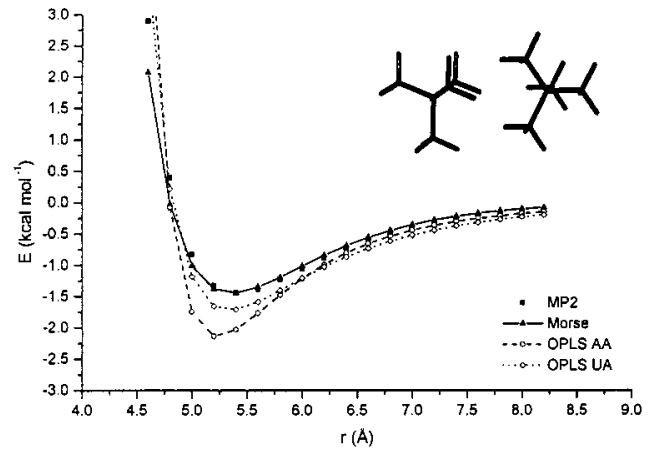

(a) Naal-Naal 90

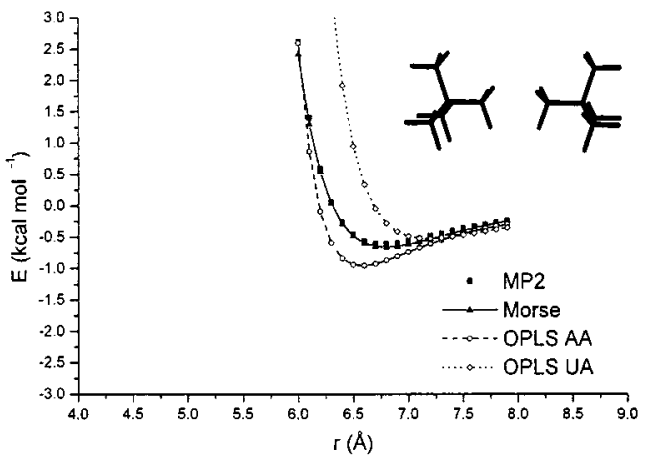

(c) Naaas-Naaas 0

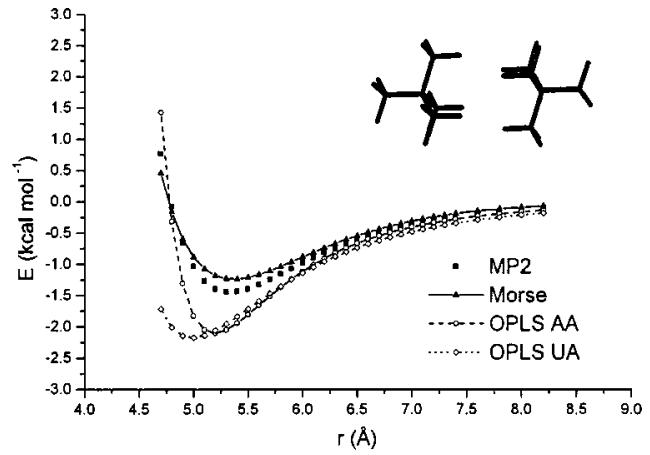

(b) Naaal-Naaal 60

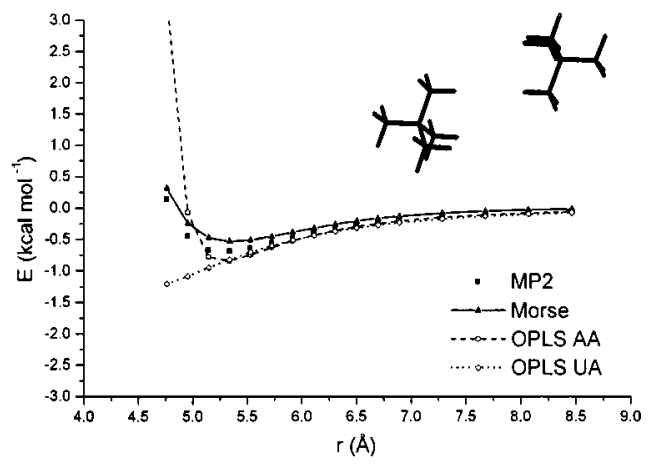

(d) $\mathrm{Na}-\mathrm{Na}$

FIG. 8. (a)-(d) Neopentane dimer potential energy surfaces from various potential models. Symbols (diamonds) represent ab initio MP2/6-311 $+\mathrm{G}(2 d f, 2 p d)$ energies, solid lines represent the energies from the pairwise additive modified Morse potential model presented here, dashed lines represent the OPLS-UA model, and dotted lines represent the OPLS-AA model.

less, the relatively good agreement of our pair potentials with empirically regressed potential models from simulations suggests that our generic pair potential models should provide good representation of the macroscopic properties when used in simulations.

\section{CONCLUSIONS}

We have shown that $a b$ initio potential energy surfaces of various small alkanes can be fitted accurately with a sum of interatomic pair potentials using a modified Morse function for each of the three constituent types of interatomic interactions. While the $\mathrm{H}-\mathrm{H}$ interactions are nearly independent of the dimer data used in the regression, the $\mathrm{C}-\mathrm{C}$ and $\mathrm{C}-\mathrm{H}$ interactions changed somewhat when data from different dimers were used in the regression. The potential energy surface for any molecule pair can be fitted more accurately independently, but simultaneous regression of parameters for the complete set of compounds studied here provides better transferability to other alkane systems. The most striking difference of the regressed modified-Morse potential models from the empirical OPLS-AA model is for the $\mathrm{C}-\mathrm{H}$ cross interaction. This is because the modified-Morse potential model presented here does not use an ad hoc relationship between like and cross parameters.

Our data set is, in its present state, heavily dominated by methyl group data and this is bound to influence the regressed parameter set. Adding more data for alkanes having non- $\mathrm{CH}_{3}$ groups in fact does slightly alter the strength of the $\mathrm{C}-\mathrm{H}$ cross interaction in particular. Comparisons of potential energy surfaces generated by the OPLS AA model with those from the $a b$ initio calculations reveal that OPLS AA potential energy surfaces are, for the most part, consistent with $a b$ initio results. Performance of our potential model in condensed phase and fine-tuning of the potential model for larger molecules are left for future work. Nevertheless, the ability of the generic parameter set to describe all of the potential energy surfaces for the ten molecular pairs of small alkanes discussed here is very encouraging.

${ }^{1}$ J.-P. Jalkanen, R. Mahlanen, T. A. Pakkanen, and R. L. Rowley, J. Chem. Phys. 116, 1303 (2002).

${ }^{2}$ D. E. Williams, J. Chem. Phys. 47, 4680 (1967).

${ }^{3}$ F. A. Momany, L. M. Carruthers, R. F. McGuire, and H. A. Scheraga, J. Phys. Chem. 78, 1595 (1974).

${ }^{4}$ A. J. Stone, The Theory of Intermolecular Forces (Oxford University Press, New York, 1996), p. 185.

${ }^{5}$ W. L. Jorgensen, J. D. Madura, and C. J. Swenson, J. Am. Chem. Soc. 106, 6638 (1984).

${ }^{6}$ A. Poncela, A. M. Rubio, and J. J. Freire, Mol. Phys. 91, 189 (1997).

${ }^{7}$ J. I. Siepmann, M. G. Martin, C. J. Mundy, and M. L. Klein, Mol. Phys. 90, 687 (1997).

${ }^{8}$ G. Kaminski, E. M. Duffy, T. Matsui, and W. L. Jorgensen, J. Phys. Chem. 98, 13077 (1994).

${ }^{9}$ W. L. Jørgensen, D. S. Maxwell, and J. Tirado-Rives, J. Am. Chem. Soc. 118, 11225 (1996)

${ }^{10}$ S. K. Nath and R. Khare, J. Chem. Phys. 115, 10837 (2001).

${ }^{11}$ H. Sun, J. Phys. Chem. B 102, 7338 (1998).

${ }^{12}$ R. L. Rowley and T. A. Pakkanen, J. Chem. Phys. 110, 3368 (1999). 
${ }^{13}$ D. H. Gay, H. Dai, and D. R. Beck, J. Chem. Phys. 95, 9106 (1991).

${ }^{14}$ T. G. Metzger, D. M. Ferguson, and W. A. Glauser, J. Comput. Chem. 18, 70 (1997).

${ }^{15}$ A. K. Rappe and E. R. Bernstein, J. Phys. Chem. A 104, 6117 (2000).

${ }^{16}$ S. Tsuzuki, T. Uchimaru, and K. Tanabe, Chem. Phys. Lett. 287, 327 (1998).

${ }^{17}$ E. Fraschini and A. J. Stone, J. Comput. Chem. 19, 847 (1998).

${ }^{18}$ J. J. Novoya, M.-H. Whangbo, and J. M. Williams, J. Chem. Phys. 94, 4835 (1991).

${ }^{19}$ S. Tsuzuki, T. Uchimaru, and K. Tanabe, Chem. Phys. Lett. 287, 202 (1998).

${ }^{20}$ S. Tsuzuki, T. Uchimaru, and K. Tanabe, J. Mol. Struct.: THEOCHEM 307, 107 (1994).

${ }^{21}$ S. Tsuzuki, T. Uchimaru, M. Mikami, and K. Tanabe, J. Phys. Chem. A 102, 2091 (1998).

${ }^{22}$ S. Tsuzuki, T. Uchimaru, K. Tanabe, and S. Kuwajima, J. Phys. Chem. A 98, 1830 (1994).

${ }^{23}$ S. Tsuzuki and K. Tanabe, J. Phys. Chem. 95, 2272 (1991).

${ }^{24}$ D. E. Williams and D. J. Craycroft, J. Phys. Chem. 91, 6365 (1987).

${ }^{25}$ R. L. Rowley, Y. Yang, and T. A. Pakkanen, J. Chem. Phys. 114, 6058 (2001).

${ }^{26}$ S. Tsuzuki, T. Uchimaru, M. Mikami, and K. Tanabe, J. Phys. Chem. A 106, 3867 (2002).

${ }^{27}$ S. Gupta, J.-A. Yang, and N. R. Kestner, J. Chem. Phys. 89, 3733 (1988).

${ }^{28}$ S. Tsuzuki, T. Uchimaru, K. Matsumura, M. Mikami, and K. Tanabe, Chem. Phys. Lett. 319, 547 (2000).

${ }^{29}$ S. Tsuzuki, T. Uchimaru, M. Mikami, and K. Tanabe, Chem. Phys. Lett. 252, 206 (1996).

${ }^{30}$ S. Tsuzuki and K. Tanabe, J. Phys. Chem. 96, 10804 (1992).

${ }^{31}$ K. Shuler and C. E. Dykstra, J. Phys. Chem. A 104, 4562 (2000).

${ }^{32}$ K. S. Kim, P. Tarakeshwar, and J. Y. Lee, Chem. Rev. 100, 4145 (2000).
${ }^{33}$ V. Spirko, O. Engkvist, P. Soldan, H. L. Selzle, E. W. Schlag, and P. Hobza, J. Chem. Phys. 111, 572 (1999).

${ }^{34}$ P. Hobza, H. L. Selzle, and E. W. Schlag, J. Am. Chem. Soc. 116, 3500 (1994).

${ }^{35}$ P. Hobza, H. L. Selzle, and E. W. Schlag, J. Phys. Chem. 97, 3937 (1993).

${ }^{36}$ P. Hobza, H. L. Selzle, and E. W. Schlag, J. Chem. Phys. 93, 5893 (1990).

${ }^{37}$ C. Gonzalez and E. C. Lim, J. Phys. Chem. A 104, 2953 (2000).

${ }^{38}$ P. Hobza, H. L. Selzle, and E. W. Schlag, J. Phys. Chem. 100, 18790 (1996).

${ }^{39}$ S. Tsuzuki, T. Uchimaru, M. Mikami, and K. Tanabe, Chem. Phys. Lett. 252, 206 (1996).

${ }^{40}$ See EPAPS Document No. E-JCPSA6-118-001309 for a more detailed explanation for selected molecules and error analysis. A direct link to this document may be found in the online article's HTML reference section. The document may also be reached via the EPAPS homepage (http:// www.aip.org/pubservs/epaps.html) or from ftp.aip.org in the directory epaps/. See the EPAPS homepage for more information.

${ }^{41}$ S. Tsuzuki, K. Honda, T. Uchimaru, M. Mikami, and K. Tanabe, J. Phys. Chem. A 103, 8265 (1999).

${ }^{42}$ W. Koch, Chemist's Guide to Density Functional Theory (Wiley VCH, Weinheim, 2000).

${ }^{43}$ M. J. Frisch, G. W. Trucks, H. B. Schlegel et al., GaUSSIAN 94, Revision E.2, Gaussian, Inc., Pittsburgh, PA, 1995.

${ }^{44}$ M. J. Frisch, G. W. Trucks, H. B. Schlegel et al., GAussian 98, Revision A.7, Gaussian, Inc., Pittsburgh, PA, 1998.

${ }^{45}$ J. Sponer and P. Hobza, Chem. Phys. Lett. 267, 263 (1997).

${ }^{46}$ R. L. Jaffe and G. D. Smith, J. Chem. Phys. 105, 2780 (1996).

${ }^{47}$ S. F. Boys and F. Bernardi, Mol. Phys. 19, 553 (1970).

${ }^{48}$ P. Blowers and R. I. Masel, J. Phys. Chem. A 103, 7725 (1999).

${ }^{49}$ J. R. Hart and A. K. Rappe, J. Chem. Phys. 97, 1109 (1992).

${ }^{50}$ W. L. Goffe, G. D. Ferrier, and J. Rogers, J. Econometr. 60, 65 (1994). 